\title{
From childhood maltreatment to adult inflammation: Evidence for the mediational status of social anxiety and low positive affect
}

\author{
Corinne N. Carlton ${ }^{\mathrm{a}, *}$, Katelyn M. Garcia ${ }^{\mathrm{a}}$, Holly Sullivan-Toole ${ }^{\mathrm{a}, \mathrm{b}}$, Kasey Stanton ${ }^{\mathrm{a}}$, \\ Christina G. McDonnell ${ }^{a}$, John A. Richey ${ }^{a}$ \\ ${ }^{\text {a }}$ Virginia Tech, Department of Psychology, USA \\ ${ }^{\mathrm{b}}$ Temple University, Department of Psychology, USA
}

\section{A R T I C L E I N F O}

\section{Keywords:}

IL-6

Social anxiety

Positive affect

Childhood maltreatment

Mindfulness

\begin{abstract}
A B S T R A C T
Prior work has established a robust association between childhood maltreatment and systemic inflammatory activation later in life; however, the mechanisms involved in this process remain incompletely understood. The purpose of this investigation was to examine potential mechanistic roles for social anxiety (SA) symptoms and low positive affect (PA) in the path from childhood maltreatment to elevations in circulating interleukin (IL)-6, a common biomarker of inflammatory activation. In addition, building on prior work establishing linkages between mindful awareness and reductions in systemic inflammation, we examined the potential role of trait mindfulness as a moderator of the relationships among childhood maltreatment, SA, low PA, and IL-6. A serial mediation model utilizing a large epidemiologic dataset (final $N=527$ ) supported our central hypothesis that the direct effect of childhood maltreatment on IL- 6 was fully serially statistically mediated by SA symptoms and low PA (but not high negative affect). Additionally, results indicated that individuals falling in the upper versus lower quartiles of SA symptoms demonstrated significantly elevated concentrations of IL-6, a finding that has not been previously reported. Trait mindfulness moderated the association between low PA and IL-6, to the exclusion of any paths related to negative affect. Additionally, results indicated that the effect of child maltreatment on IL-6 bypasses SA to indirectly impact IL-6 via negative affect. Overall, we conclude that childhood maltreatment and SA symptoms have a significant influence on IL-6, albeit indirectly via low PA, and the influence of PA on IL-6 may be uniquely susceptible to influence by individual differences in mindfulness.
\end{abstract}

\section{Introduction}

Childhood maltreatment refers to experiences of abuse within physical, sexual, or psychological domains, as well as physical or emotional neglect, and is related to negative psychological and physical health outcomes across the lifespan (Barnett et al., 1993; Cicchetti, 2016; Cicchetti and Toth, 2005). A range of biological mechanisms have been previously proposed to explain how early adverse experiences such as childhood maltreatment predict negative health sequelae, and have focused largely on chronic inflammatory activation as an explanatory model (Baumeister et al., 2016; Hertzman and Boyce, 2010; Holochwost et al., 2020). However, the mechanisms by which childhood maltreatment culminates in chronic inflammatory involvement in adulthood remain incompletely understood. Significant prior work has explored the possibility that childhood maltreatment may confer vulnerability to systemic inflammation specifically through suppression of neural systems that subserve reward-related processes (Danese and Lewis, 2017; Dantzer et al., 2008; Mehta et al., 2020; Miller et al., 2009; Nusslock and Miller, 2016). This broader body of work therefore highlights the possibility that childhood maltreatment may trigger systemic inflammation later in life through its connection with affective disorders that involve anhedonia, or loss of interest or pleasure toward stimuli that were previously perceived as rewarding (American Psychiatric Association, 2013). Whereas most work exploring connections between anhedonia and inflammation has focused on depression (Danese et al., 2008), more recent work has highlighted that social anxiety comprises an anhedonic component (e.g., Kashdan et al., 2011). Additionally, recent work has indicated that anhedonia within social anxiety is observed more frequently amongst adult survivors of childhood maltreatment (e.g., Simon et al., 2009). It therefore stands to reason that social anxiety may be both an affective consequence of childhood maltreatment and also may mediate the evolution of inflammatory biomarkers due exclusively

\footnotetext{
* Corresponding author. Virginia Tech, Department of Psychology, 109 Williams Hall, Blacksburg, VA, 24061, USA.

E-mail address: corinnecarlton@vt.edu (C.N. Carlton).
} 
to its association with low positive affect (PA) - a central component of anhedonia (Craske et al., 2016) - among individuals high in social anxiety. Accordingly, the purposes of the current study were to (1) probe the existence of an overall association between social anxiety symptom severity and concentrations of the circulating proinflammatory cytokine interleukin (IL)-6 in a population-based sample and (2) to test a theoretical model involving high social anxiety and low PA as serially positioned mediators of the association between childhood maltreatment and elevated IL-6 in adults. We further sought to examine (3) an exploratory question involving the moderating role of trait mindfulness, which has been previously linked to suppression of inflammatory biomarkers including IL-6 (Creswell et al., 2016).

Child maltreatment has been associated with significant mental health consequences in adulthood, including affective disorders such as depression and anxiety (Gardner et al., 2019). Anxiety disorders, in turn, have been strongly and consistently linked to chronic inflammatory activation in adult samples (Michopoulos et al., 2017; Vogelzangs et al., 2013). Among the DSM-5 defined anxiety disorders, social anxiety disorder (SAD) consistently ranks among the most common anxiety diagnoses in both adult (Kessler et al., 2005) and youth samples (Merikangas et al., 2010). However, despite its prominence within the broader class of anxiety disorder diagnoses, surprisingly little research has considered the pathways and mechanisms by which social anxiety symptomatology might be related to inflammatory involvement. It has however been previously demonstrated that adults endorsing high levels of social anxiety symptoms report a significantly higher frequency of childhood emotional abuse, emotional neglect and sexual victimization than adults with lower levels of social anxiety symptoms (e.g., Bandelow et al., 2004; Bruce et al., 2013; Kuo et al., 2011), which therefore bolsters the idea that this population may be at particular risk for systemic inflammation. Data from adults with social anxiety reported by Simon et al. (2009) indicated that nearly $70 \%$ of adults with diagnosed SAD endorsed at least one form of childhood maltreatment on the Childhood Trauma Questionnaire (CTQ; Berstein and Fink, 1998), and CTQ scores in this sample were further correlated with overall social anxiety symptom severity. Nearly identical findings were later reported by Kuo et al. (2011), who demonstrated significantly higher endorsed frequencies of childhood maltreatment among adults diagnosed with SAD, results which have been supported across a range of related studies (e.g., Bandelow et al., 2004; Kessler et al., 1997; Taillieu et al., 2016). Taken together, these findings collectively suggest that childhood maltreatment may serve as a common linkage for both social anxiety symptomatology as well as inflammatory activation in adulthood.

Although the specific means by which early adverse experiences lead to inflammatory involvement in adulthood remains an active area of inquiry, the presence of a strong association between these scenarios has been increasingly appreciated. For example, prior work has established that childhood maltreatment leads to abnormalities in stress-related neurobiological systems, which in turn is thought to influence both immune and inflammatory activation (Danese et al., 2007, 2008; Danese and Lewis, 2017). In their seminal work on this topic, Danese et al. (2007) established that childhood maltreatment acts as a distinct and potentially unique risk factor for the development of inflammation in adulthood. This association was further evaluated among depressed adults, to evaluate whether childhood maltreatment alone could identify depressed individuals with co-occurring elevated inflammation levels (Danese et al., 2008). Results from this investigation identified that childhood maltreatment predicted higher rates of inflammation in individuals experiencing depression, suggesting that childhood maltreatment is associated with the co-occurrence of depression and inflammation. In addition to linkages between childhood maltreatment and inflammation, recent work has further indicated this variation in stress-related neural systems as a susceptibility factor for the development in anhedonia (which comprises a significant deficit in PA) later in life, largely due to associated reward system suppression (for review, see Swardfager et al., 2016).
In considering psychologically relevant paths and mechanisms toward systemic inflammation, increased concentrations of peripheral circulating cytokines have been previously linked to both increased negative emotionality as well as reduced positive emotionality (e.g., Graham-Engeland et al., 2018; Sturgeon et al., 2016). Evidence further suggests that lower levels of PA influence inflammatory processes via mechanisms that may be distinct from high levels of negative affect ([NA]; Fancourt and Steptoe, 2020; Prather et al., 2007; Slavish et al., 2020; Steptoe et al., 2008). Studies involving non-clinical samples have previously highlighted the association between variations in PA with inflammation levels. For example, in samples of young and middle-aged adults, diminished PA has been linked to lower levels of IL- 6 and other markers of inflammation, but not NA (Ong et al., 2018; Stellar et al., 2015). Additionally, higher aggregated levels of positive mood, measured via ecological momentary assessments, have been associated with reduced levels of inflammatory markers including C-reactive protein and IL-6 in women, although not in men (Steptoe et al., 2008). Similarly, individuals reporting a greater frequency of daily positive events had lower levels of IL-6, with the most pronounced effects in participants within the lowest quartile of reported positive event frequency (Sin et al., 2015). However, it should be noted that the literature regarding the association between PA and inflammation is mixed, with some studies reporting weak and/or null findings in the relation between PA and circulating IL-6 (e.g., Andreasson et al., 2013; Carroll et al., 2011). For example, Andreasson et al. (2013) identified that the absence of PA was weakly associated with IL-6. Moreover, Carroll et al. (2011) specifically examined changes in PA as they related to the magnitude of IL-6 responses. Findings from their study suggested that changes in PA were not related to IL- 6 responses. Despite these findings, many other studies (as noted above) have indeed demonstrated a linkage between PA and inflammatory activity. However, it should be further emphasized that there are other studies that find small effects (e.g., Andreasson et al., 2013), and null findings are also common (e.g., Carroll et al., 2011; Friedman et al., 2007).

The majority of available literature probing the interface between affect and inflammation has focused on distress-driven relationships (e.g., Kiecolt-Glaser et al., 2002) which has reinforced the role of psychological stress in inflammatory activation and the role of negative affect in levels of circulating cytokines (Steptoe et al., 2007; Marsland et al., 2017; Sutin et al., 2010). Detailed mechanisms have been characterized within the nuclear transcription factor kappa-light-chain-enhancer of activated B cells (NF-kB) signaling pathway, which plays a central role in inflammatory disease through its ability to induce transcription of proinflammatory genes (Tak and Firestein, 2001). NF-kB is found in virtually every cell of the immune system, and regulates multiple aspects of innate and adaptive immune responses by up-regulating transcriptional factors of inflammatory mediators in different types of immune cells (Liu et al., 2019). In particular, this pathway is thought to be involved in translating the effects of psychosocial stress into cellular activation (Bierhaus et al., 2003) by inducing transcriptional induction of proinflammatory cytokines including but not limited to IL-1, IL-6, IL-1 $\beta$ and tumor necrosis factor alpha (TNF- $\alpha$; Lawrence, 2009). For example, Kuebler et al. (2015) demonstrated that acute psychological stress induced rapid increases in NF-kB DNA binding activity (1 to $10 \mathrm{~min}$ post stressor), as well as deployed increases in both mRNA and plasma levels of IL- 6 and IL-1 $\beta$ (approximately 120 min post stressor).

Recently however, a small but growing literature has explored the possibility that either in addition to or possibly even separate from stressrelated elevations in NA, decreases in positive affect may also be linked to inflammatory activation (Steptoe et al., 2007a,b; Matthews et al., 2010; Ong et al., 2018). Slavish et al. (2020) recently found that after controlling for a range of related factors including age, gender and BMI, higher PA was associated with lower salivary C-reactive protein (CRP; although not salivary IL-6), among young adults even after controlling for NA. This is largely consistent with earlier work from Stellar et al. (2015), 
who found that trait positive affect was concurrently associated with lower levels of IL-6. This is further consistent with experimental work from Hunt et al. (2021), who demonstrated that trait PA moderates the associations between sleep disruption and stimulated monocyte production of IL-6, independent of negative affect. Likewise, Prather et al. (2007) reported that individuals higher in PA showed decreased in vitro production of IL- 6 and IL-10 in response to stimulation with endotoxin. Steptoe, Wardle and Marmot (2005) similarly reported that PA was significantly associated with lower levels of plasma fibrinogen, an inflammatory marker and predictor of future heart disease. In the diagnostic realm, Kudinova et al. (2020) recently reported data from adults with or without a history of recurrent depression (rMDD), and found that current anhedonia rather than rMDD was associated with lipopolysaccharide-stimulated levels of inflammatory markers. This suggests that inflammatory activation may be uniquely associated with anhedonia rather than recurrent depression more generally. Thus, the roles of psychological wellbeing and positive emotions are increasingly recognized in terms of their potential unique influence over inflammatory pathways that are, in turn, related to significant eventual health outcomes.

In further considering the directionality of pathways observed in this correlational work (i.e. does inflammation cause suppression of positive emotions or vice versa?), it has been experimentally demonstrated that paradigms using exogenously-induced inflammation provide evidence for a specific directional effect of cytokine elevations, wherein proinflammatory cytokines may serve as mediators of environmental and neurobiological factors that contribute to the development of depression (Felger and Miller, 2012; Felger and Lotrich, 2013). Significant experimental work in animals (e.g. Rossetti et al., 2016; see also De La Garza 2005) and humans (Eisenberger et al., 2010; Bell et al., 2017) have supported the existence of a causal relationship between exogenous up-regulation of circulating proinflammatory markers (typically via endotoxin; DellaGioia and Hannestad, 2010) and anhedonic symptoms, which may themselves be related to low PA (Janicki-Deverts et al., 2007). However, a separate (bi-) directional causal effect has been theorized elsewhere (Kiecolt-Glaser et al., 2015, in which depression may prime cytokine responses to stressors that do not habituate (Copeland et al., 2012; Deverts et al., 2010; Duivis et al., 2011; Euesden et al., 2017). The mechanistic pathways involved in this directional effect remain an ongoing topic of research, but may involve dopamine (DA)-driven regulation of the activity, migration, differentiation and proliferation of immune cells (Beck et al., 2004). Overwhelming evidence suggests that a primary function of DA in the central nervous system and mesolimbic DA system in particular is to determine motivational drive (e.g. Bromberg-Martin, et al., 2010). Anhedonia and low PA are associated with low motivational drive and lowered synaptic DA availability (e.g. Dunlop and Nemeroff, 2007; Yadid and Friedman, 2008), and may therefore play a role up-regulating CNS inflammatory activation. DA receptors are expressed on immune cells including $\mathrm{T}$ and B lymphocytes (Santambrogio et al., 1993), dendritic and NK cells, macrophages and neutrophils among others (Arreola et al., 2016). Thus, in addition to its role as a neurotransmitter, DA is also a peripheral chemical mediator that controls systemic inflammation, likely via its indirect influence over cytokine networks including IL-6 (Beck et al., 2004) and possibly also by inhibiting nuclear translocation of the p65 subunit of NF- $\mathrm{kB}$ (Yoshioka et al., 2020). Thus, the influence of positive emotionality on inflammatory activation may be distinctly regulated by DA which is in turn known to play a role in motivational drive, although the precise mechanisms by which this occurs awaits future research. It should also be noted however, that heightened inflammatory cytokines also reduce the availability of DA (e.g., Felger, 2016), thus highlighting the potential bidirectional nature of this mechanism. In the current study however, the motivation to examine the directional influence of positive emotion on inflammatory involvement stems from the need to identify modifiable predictors of negative health sequelae that may be engaged by psychological interventions. Indeed, a central justification for examining this particular direction in a bi-directional system lies in its potential to instantiate potentially modifiable targets into a conceptual framework for future psychologically-based intervention efforts.

The prior work outlined above has identified the potential influence of low PA in the path to inflammation and offers a theoretical framework for understanding the social anxiety-inflammation interface, as it is increasingly accepted that SAD comprises a significant PA deficit (Brown et al., 1998; Kashdan, 2007; for review see Richey et al., 2019). Deficiencies in PA associated specifically with social anxiety are manifested in a variety of ways. For example, on a daily basis, individuals with symptoms of social anxiety report fewer positive emotions and positive events, report less time feeling happy and relaxed, and report less intense positive emotional experiences (Kashdan and Collins, 2010; Kashdan and Steger, 2006). As attenuated PA in social anxiety cannot be explained by depressive symptoms (Kashdan, 2007) and is not characteristic of other anxiety disorders (Brown et al., 1998), blunted PA stands as a distinguishing feature of SAD. Therefore, given the correspondence between social anxiety diminished PA, it follows that elevated levels of circulating cytokines would be found among individuals endorsing high social anxiety symptomatology and low PA.

Taken together, the extant literature characterizing the nexus of childhood maltreatment, anhedonia, inflammation and social anxiety symptoms collectively suggest that the long-term consequences of early adversity include significant risk for social anxiety symptoms as well as exaggerated expression of proinflammatory cytokine responses in adulthood. Within this line of reasoning, it also follows that social anxiety symptomatology, is (1) a potential consequence of childhood maltreatment and (2) is linked to low PA, and may therefore play a unique role in the pathway from childhood maltreatment to inflammation-related health consequences in adulthood. However, despite the clear theoretical link between childhood maltreatment, social anxiety symptoms and inflammatory involvement, no study has yet characterized patterns of IL6 among adults with social anxiety symptomatology, nor has it been determined whether this effect may instead be attributable to the presence of core anhedonic symptoms such as low PA. One notable exception however, is found in one study examining the link between IL-2 (another form of proinflammatory cytokine) and social anxiety symptoms, which supported the existence of a significant association, consistent with our general series of predictions here (Rapaport and Stein, 1994). However, with the exception of this one study, no prior work has systematically examined pathognomonic risk and resilience factors specific to SAD that may influence its association with inflammation, an area of inquiry with translational potential for identifying protective factors that may mitigate the negative biological sequelae of affective pathology. Lastly, an emergent body of work has provided initial support for the role of mindfulness as a mitigating factor in the path from stress to inflammatory activation (Malarkey et al., 2013; Rosenkranz et al., 2013; Sanada et al., 2020; Villalba et al., 2019). A conceptually related body of work has further postulated that anhedonia in particular may be both a consequence of psychological stress, a cause of systemic inflammation (Rossetti et al., 2016), and may also be susceptible to the moderating influence of mindfulness (Sanada et al., 2020; Villalba et al., 2019). In support of this general model, recent evidence has demonstrated that increases in neurofunctional markers of reward activation were correlated with decreases in circulating inflammatory markers in a single-armed mindfulness intervention (Dutcher et al., 2020). As such, mindfulness may play a plausible role in moderating the association of anhedonic markers with inflammatory activation, which may hold promise for the development of interventions aimed at preventing its negative health sequelae.

Accordingly, the first aim of this investigation was to examine the robustness of the association between social anxiety symptoms and concentrations of the circulating proinflammatory marker IL-6. Second, we sought to test the hypothesis that the association between childhood 
maltreatment and IL- 6 may be mediated by social anxiety and features of anhedonia, particularly low PA. We specifically hypothesized that low PA, which has been established through prior work as a significant predictor of inflammation (e.g., Ong, et al., 2018; Stellar et al., 2015), as well as a specific consequence of social anxiety symptoms (Kashdan, 2007; Richey et al., 2019), would therefore mediate the direct effect of social anxiety on IL-6. We further anticipated that childhood maltreatment, which is among the most robust predictors of both social anxiety symptomatology (Kuo et al., 2011; Simon et al., 2009) and chronic inflammation in adulthood (Baumeister et al., 2016), would influence IL-6 levels albeit indirectly via social anxiety and low PA.

In addition, trait mindfulness has also emerged as a variable of particular interest in this line of inquiry, as prior research has consistently indicated that inflammatory markers are engaged and perhaps directly modulated by intra-individual differences in mindful awareness (e.g., Ng et al., 2020; Rosenkranz et al., 2013). Additionally, recently trait mindfulness has been identified as a potential buffer to the adverse consequences of childhood maltreatment (e.g., Beshai \& Parmar, 2019). Moreover, trait mindfulness has shown negative associations with both high social anxiety (e.g., Makadi and Koszycki, 2020) as well as with self-reports of low PA (Jislin-Goldberg et al., 2012). Therefore, given these distinct associations the present study also explored the possibility that trait mindfulness moderates the associations among childhood maltreatment, social anxiety, PA and IL-6.

\section{Method}

\subsection{Data}

We used publicly available data from the Midlife In the United States (MIDUS) project, a national multi-site longitudinal study of health and well-being (https://midus.wisc.edu; Radler, 2014). Data reported here were drawn from a subset of the first follow-up on the original sample (MIDUS-2 - Biomarkers Project), which was collected between 2004 and 2009. Of the individuals who participated in the MIDUS-2 Biomarkers study, 1054 had complete data from the main survey session as well. Of these 1054 participants, 728 (Female $=361$, Male $=367$ ) had complete data for all dependent and independent measures of interest. Participants in this subsample were between the ages of 26 and 78 years $\left(\mathrm{M}_{\mathrm{Age}}=53.53 ; \mathrm{SD}=13.68\right)$. On average, data for the biomarker session were collected 25.85 months after the survey session (SEM Lag $=.460$ months). The MIDUS-2 survey (collected between 2004 and 2006) used the same comprehensive assessments (e.g., cognitive assessments; daily stress diaries) as those in the original longitudinal MIDUS sample with additional economic questions included (e.g., questions relevant to SES). Cognitive and socioemotional questionnaires were administered and then biomarker and neuroscience assessments were also gathered on a subsample of the MIDUS- 2 respondents. This was done in order to facilitate work probing biological influences on behavioral outcomes and vice versa as described in the MIDUS-2 Biomarkers Project (such as IL-6, in the current study). In addition to requiring complete data on all dependent and independent measures of interest, we further eliminated cases who did not have complete data on all covariates of interest (e.g. SSRI use, BMI as described in detail further below) within the combined datasets. This subset of respondents $(\mathrm{N}=527)$ was comparable to the initial sample on sociodemographic factors (age, gender, marital status, and education). Participants in this final subsample were between the ages of 26 and $78\left(\mathrm{M}_{\mathrm{Age}}=53.23\right.$; $\left.\mathrm{SD}=13.43\right)$. Of the 527 participants that had complete data for the present study, $82.7 \%$ identified as White/Caucasian, 7.6\% identified as Black/African American, 2.1\% identified as Native American or Alaska Native/Aleutian Islander/Eskimo, 0.9\% identified as Asian, and 5.9\% identified as Other. Additionally, $0.8 \%$ of the sample reported either "don't know" or opted to not answer how they racially identified. Table 1 contains additional demographic information on the final sample.
Table 1

Demographic breakdown.

\begin{tabular}{lll}
\hline & Mean & SD \\
\hline Age $(\mathbf{n}=\mathbf{5 2 7})$ & 52.23 & 13.43 \\
Sex & $\mathbf{N}$ & Percent \\
$\quad$ Female & 269 & $51 \%$ \\
Male & 258 & $49 \%$ \\
Racial Origin & & \\
$\quad$ White & 436 & $82.7 \%$ \\
African American & 40 & $7.6 \%$ \\
Native American & 11 & $2.1 \%$ \\
Asian & 5 & $0.9 \%$ \\
Other & 31 & $5.9 \%$ \\
Don't know & 2 & $0.4 \%$ \\
No response & 2 & $0.4 \%$ \\
Wage & & \\
Less than $\$ 0-13,999$ & 180 & $34.2 \%$ \\
$\$ 14,000-\$ 24,999$ & 41 & $7.8 \%$ \\
$\$ 25,000-\$ 34,999$ & 43 & $8.2 \%$ \\
$\$ 35,000-\$ 49,999$ & 48 & $9.1 \%$ \\
$\$ 50,000-\$ 69,999$ & 70 & $13.3 \%$ \\
$\$ 70,000-\$ 89,999$ & 39 & $7.4 \%$ \\
$\$ 90,000-\$ 129,999$ & 39 & $7.4 \%$ \\
$\$ 130,000-\$ 174,999$ & 18 & $3.4 \%$ \\
$\$ 175,000-\$ 249,999$ & 7 & $1.3 \%$ \\
$\$ 300,000$ or more & 2 & $0.4 \%$ \\
No response & 40 & $7.6 \%$ \\
\hline
\end{tabular}

\subsection{Covariates and exclusionary criteria}

Exclusionary criteria and relevant covariates were based on the clear guidelines provided by O'Connor et al. (2009) regarding the effects of biobehavioral variables on circulating proinflammatory markers, further detailed as follows. Specifically, we included the following as covariates in all of the reported models: 1) Sex, 2) age at clinic visit, 3) waist-to-hip ratio, 4) highest level of education completed, 5) current SSRI use [yes/no], 6) current antihypertensive use [yes/no], 7) current regular exercise or activity of any type for $20 \mathrm{~min}$ or more at least 3 times per week. [yes/no]. In addition, we excluded cases who answered yes to any of the following criteria: 1) personal history of cancer [yes/no], 2) personal history of chemotherapy [yes/no], 3) current insomnia or sleep disorder [yes/no], 4) personal lifetime history of drug or alcohol problem [yes/no], 5) personal history of diabetes [yes/no]. It should lastly be noted that we were not able to covary or exclude cases endorsing tobacco use due to excessive missing data on this variable in the MIDUS database, such that $>50 \%$ of cases lacked information about current tobacco use.

\subsection{Measures}

Descriptive statistics for all measures involved in the present study are displayed in Table 2.

\subsection{Primary self-report measures}

Childhood Trauma Questionnaire (CTQ; Berstein and Fink, 1998). Participants completed the CTQ, a 28-item questionnaire that retrospectively assesses childhood maltreatment experiences. This measure

Table 2

Descriptive statistics for all variables of interest.

\begin{tabular}{lll}
\hline & Mean & SD \\
\hline IL-6 & .28 & .35 \\
Childhood Maltreatment & 38.08 & 13.70 \\
Social Anxiety & 1.87 & .54 \\
Positive Affect & 3.37 & .74 \\
Negative Affect & 1.52 & .65 \\
Mindfulness & 33.16 & 7.71 \\
\hline
\end{tabular}


includes five factors: emotional abuse, physical abuse, sexual abuse, emotional neglect, and physical neglect. Participants rated how often they experienced each item when they were a child and/or a teenager on a Likert scale, ranging from 1 ("Never True") to 5 ("Very Often True"). We used the total sum of items across all five subscales to assess for the general presence of childhood maltreatment. Cronbach's alpha for this scale was acceptable across subscales in the MIDUS-R sample (total scale $\alpha=.902$; emotional abuse $\alpha=.860$; physical abuse $\alpha=.800$; sexual abuse $\alpha=.946$, emotional neglect $\alpha=.897$, physical neglect $\alpha=.678$ ).

Social Anxiety Scale (SAS; Fresco et al., 2001). The SAS is a 9-item questionnaire that assesses severity of social anxiety symptoms. Participants were asked to rate how much fear or anxiety they generally feel across various social situations (e.g., "talking with people you don't know very well") on a 4-point Likert scale, ranging from 1 ("None") to 4 ("Severe"). We computed the final scale score using the standardized procedure of computing the mean across all scale items. Higher scores on this scale are indicative of greater social anxiety symptom severity. This scale showed good internal consistency across the MIDUS-R subsample (Cronbach's $\alpha=.852$ ).

Positive and Negative Affect Schedule (PANAS; Watson et al., 1988). The PANAS is a 20 -item questionnaire that assesses current positive and negative affect. These 20 items consist of 10 adjectives that describe positive feelings/emotions and 10 adjectives that describe negative feelings/emotions. Participants were asked to rate the extent to which each word describes how they are currently feeling using a 5-point Likert scale, ranging from 1 ("Very slightly or not at all) to 5 ("Extremely"). Higher scores on the scales are indicative of high positive or negative affect. Cronbach's alpha for this questionnaire was good (positive affect scale: $\alpha=.863$; negative affect scale $\alpha=.829$ ).

Trait Mindfulness (Langer and Moldoveanu, 2000). A 9-item self-administered questionnaire assessing different questions related to the construct of mindfulness was used to calculate level of mindfulness. Participants were asked to respond to questions on a 5-point Likert-type scale ranging from "strongly agree" to "strongly disagree". The total score for this scale was calculated as a sum of all items, and all items were reverse coded such that higher scores indicate a higher amount of mindfulness. Internal consistency for the scale was good (Cronbach's $\alpha=$ .957).

\subsection{Inflammatory markers}

Following a 12-h fasting period, blood samples were collected from each participant using standardized procedure. Levels of IL-6 were analyzed and quantified per participant through standard ELISA methodology. Both Kolmogorov-Smirnov and Shapiro-Wilk tests of normality suggested significant deviations from the normal distribution for all markers (all positively skewed; all $p s<.001$ ). Qualitative inspection of normal and detrended Q-Q plots and stem-and-leaf plots provided evidence for considerable positive skew for IL-6, which were thus natural $\log$ transformed, consistent with common practice. Post-transformation, plots were visually inspected to ensure normal distribution.

\subsection{Statistical approach}

All relevant variables of interest were first correlated, and then tests of mediation were conducted using Hayes's (2012) PROCESS macro for SPSS. Various measures of effect size are available for direct, indirect and total effects, along with bootstrapped confidence intervals for effect size inference within PROCESS. PROCESS is computationally distinct from structural equation modeling (SEM) frameworks, although prior work has demonstrated that SEM will produce generally identical results to those produced by PROCESS (Hayes et al., 2017), particularly when all variables are observed and not latent, as is the case here. In order to test hypotheses related to associations between social anxiety symptomology and IL-6, as well as the influence of risk factors such as childhood maltreatment, PA and NA on this association, we implemented a serial mediation model (PROCESS model 6; Fig. 2) with PA and high social anxiety as potential mediators in the relationship between childhood maltreatment and IL-6 (PROCESS model 6). We also evaluated a more stringent model controlling for NA, in order to examine hypothesized patterns among theoretically relevant variables while accounting for NA as a covariate over all paths. Similarly, an additional serial mediation model was run, substituting NA instead of PA to assess differential mediational effects (i.e. substituting NA for PA as the second serially positioned mediator, using the same structural model). We also evaluated the influence of PA as a covariate over our primary hypothesized model. Additionally, in order to test hypotheses regarding the role of mindfulness as a potential moderator in the relationship between childhood maltreatment, social anxiety, low PA and IL-6 we ran an exploratory model (moderated mediation or conditional indirect effects) exploring the potential moderating effect of mindfulness on all paths in the model simultaneously (PROCESS model 92; Fig. 3). As above, an additional moderated-mediation model was run for with NA instead of PA to probe differential effects.

\section{Results}

\subsection{Overview of analytic strategy}

Pearson correlations were computed among measures of interest to establish initial associations among theoretically relevant variables (results shown in Table 3). Next, associations between social anxiety symptoms and log-transformed IL-6 were examined through linear regression analyses. The full model evaluated serial mediation with PA and social anxiety as potential mediators in the association between childhood maltreatment and IL-6 (PROCESS model 6). An additional serial mediation model was run for with NA instead of PA to determine differential effects (PROCESS model 6). To probe the third aim of this study, we implemented a moderated-mediation model (probing moderation simultaneously over all mediated paths) to explore the potential role of mindfulness as a moderator of the relationship between childhood maltreatment, IL-6, social anxiety and PA (PROCESS model 92). Specifically, this moderated-mediation approach will demonstrate if there is an interaction effect of mindfulness over one of the mediated paths in the relation between variables of interest. To probe comparative effects related to NA, we implemented an identical additional moderatedmediation model, with the sole exception of substituting NA for PA as the second serially positioned hypothesized mediator, in order to establish differential effects of NA versus PA in the proposed models.

\subsection{Correlations}

As expected, and consistent with prior work, correlational results revealed positive associations between IL- 6 and childhood maltreatment $(r=.12, p<.01)$, social anxiety and childhood maltreatment $(r=.15$, $p<.001)$, IL-6 and NA $(r=.10, p<.05)$, childhood maltreatment and NA $(r=.23, p<.001)$, social anxiety and NA $(r=.30, p<.001)$, and PA and trait mindfulness $(r=.13, p \leq .001)$. Additionally, negative correlations were observed for PA with IL-6, childhood maltreatment, and social anxiety $(r=-.09, p<.05 ; r=-.25, p<.001 ; r=-.27, p<.001$, respectively) as well as NA and PA $(r=-.63, p<.001)$. Surprisingly however, the social anxiety scale measure demonstrated no significant relationship with IL- 6 in the total sample $(r=-.07, p>.05)$. We therefore followed up with a series of exploratory analyses, which first probed the possibility of a non-linear relationship between these two variables. Accordingly, we first probed the quadratic term in a GLM framework, which supported the existence of a nonlinear relationship (linear model results $\beta=-.02, p=.212$; quadratic model results $\beta 1=-.04, \beta 2=.05$, $p<.01$; see Fig. 1) between social anxiety and IL-6, such that the upper quartile of social anxiety symptom severity was significantly correlated with IL-6 $(r=.24, p<.05)$, whereas the lower quartile was not $(r=-.06$, $p=.59$ ). Further, although no linear effect of social anxiety symptoms of 
Table 3

Correlations between all variables of interest and covariates.

\begin{tabular}{|c|c|c|c|c|c|c|c|c|c|c|c|c|c|}
\hline & 1 & 2 & 3 & 4 & 5 & 6 & 7 & 8 & 9 & 10 & 11 & 12 & 13 \\
\hline 1. IL-6 & - & & & & & & & & & & & & \\
\hline 2. Childhood Maltreatment & $.12 * *$ & - & & & & & & & & & & & \\
\hline 3. Social Anxiety & -.07 & $.15^{* * *}$ & - & & & & & & & & & & \\
\hline 4. Positive Affect & $-.09 *$ & $-.25^{* * *}$ & $-.27^{* * *}$ & - & & & & & & & & & \\
\hline 5. Negative Affect & $.10^{*}$ & $.23^{* * *}$ & $.30^{* * *}$ & $-.63^{* * *}$ & - & & & & & & & & \\
\hline 6. Mindfulness & .01 & .01 & .07 & $.13^{* * *}$ & .01 & - & & & & & & & \\
\hline 7. Sex* & -.03 & $-.14 * *$ & $.08^{*}$ & .04 & .01 & $.20 * *$ & - & & & & & & \\
\hline 8. Age* & $.42 * *$ & -.04 & $-.20 * *$ & $.13^{* *}$ & $-.18^{* *}$ & -.02 & $-.09 *$ & - & & & & & \\
\hline 9. Waist-Hip Ratio* & $.31 * *$ & -.04 & $-.09 *$ & -.04 & -.01 & $-.13^{* * *}$ & $-.63^{* *}$ & $.29 * *$ & - & & & & \\
\hline 10. Education Level* & $-.17 * *$ & $-.16^{* *}$ & -.06 & .07 & $-.16 * *$ & -.07 & -.03 & .01 & $-.11 * *$ & - & & & \\
\hline 11. SSRI Status* & -.06 & -.07 & $-.08 *$ & $.15^{* *}$ & $-.12^{* * *}$ & -.02 & $-.08 *$ & .01 & .01 & -.01 & - & & \\
\hline 12. Antihypertensive Status* & $-.35 * *$ & .01 & $.13^{* *}$ & -.01 & .02 & .02 & $.09 *$ & $-.43^{* *}$ & $-.31 * *$ & .04 & -.03 & - & \\
\hline 13. Exercise* & .14 & .02 & .04 & $-.08 *$ & .07 & -.03 & -.00 & -.01 & $.13^{* *}$ & $-.16^{* *}$ & -.07 & -.00 & - \\
\hline
\end{tabular}

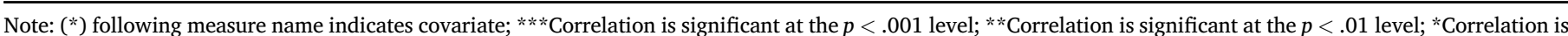
significant at the $p<.05$ level.

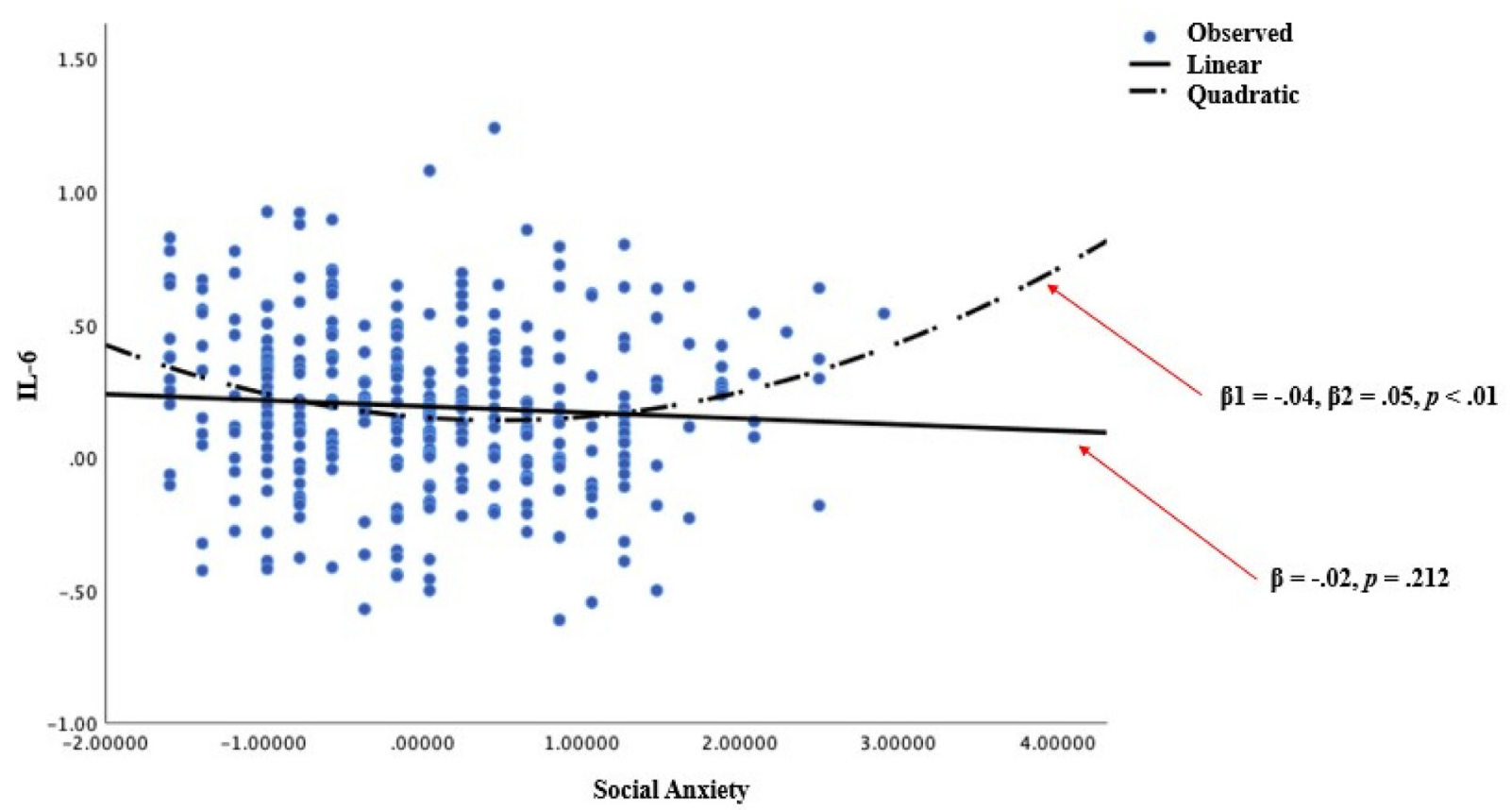

Fig. 1. Demonstration of non-linear relationship between IL-6 and social anxiety.

IL- 6 was observed in the total sample $(F(1,319)=1.563, p=.212)$, cases falling in the upper (but not lower) quartile of social anxiety symptoms demonstrated significantly elevated concentrations of IL-6 (upper quartile: $\beta=.28, \mathrm{p}<.05$; lower quartile: $\beta=-.18, \mathrm{p}=.593$ ), which is consistent with the nonlinear effect observed in the total sample.

\subsection{Mediation models}

Our principal hypothesized model evaluated mediation of childhood maltreatment on IL-6, by social anxiety symptoms and PA as potential serially positioned mediators (PROCESS model 6) with NA as a control variable. Results of this comparatively more stringent model suggested that the direct effect of childhood maltreatment on IL-6 was not significant $(\mathrm{b}=.001, \mathrm{p}=.21)$. Within this approach, childhood maltreatment was not significantly associated with social anxiety $(\mathrm{b}=.020, \mathrm{p}=.25)$ but was significantly related to PA $b=-.007, \mathrm{p}<.001$ ). In light of the diminished direct effect of childhood maltreatment on IL-6 in contradiction to prior work (e.g., Kiecolt-Glaser et al., 2011), it is possible that this pattern of results may be due to the strong (anti) correlation between PA and NA (-0.63 in our data), such that comparatively sparse variance remained to drive the hypothesized associations. We therefore considered a separate model presented here, that does not control for NA (see Fig. 2). Results from this model indicated that childhood maltreatment was significantly and concurrently associated with both high social anxiety and low PA $(b=.005, p<.01 ; b=-.013, p<.001$, respectively). The direct effect of childhood maltreatment on IL- 6 was initially significant, prior to considering potential indirect or mediating effects $(b=$ $.002, p<.05$ ); however, consistent with the existence of full mediation, the direct effect of childhood maltreatment on IL-6 was no longer significant after accounting for the presence of the hypothesized mediators ( $b=.002, p=.14$ ). Further consistent with the presence of serial mediation, childhood maltreatment predicted social anxiety $(b=.005$, $p<.01)$, social anxiety next predicted PA $(b=-.338, p<.001)$, and PA lastly predicted IL- $6(b=-.058, p<.01)$ in the mediational model. No partial indirect effects were observed (i.e. single mediation of CTQ on IL-6 by social anxiety only or PA only). Thus, results from this model provided evidence for full serial mediation of the effect of child maltreatment on IL- 6 by social anxiety symptoms and low PA.

To probe the possible differential role of NA in the model structure described above, an additional serial mediation model was run substituting NA for PA as the second serially positioned mediator (PROCESS model 6). As was the case with the initial stringent model 


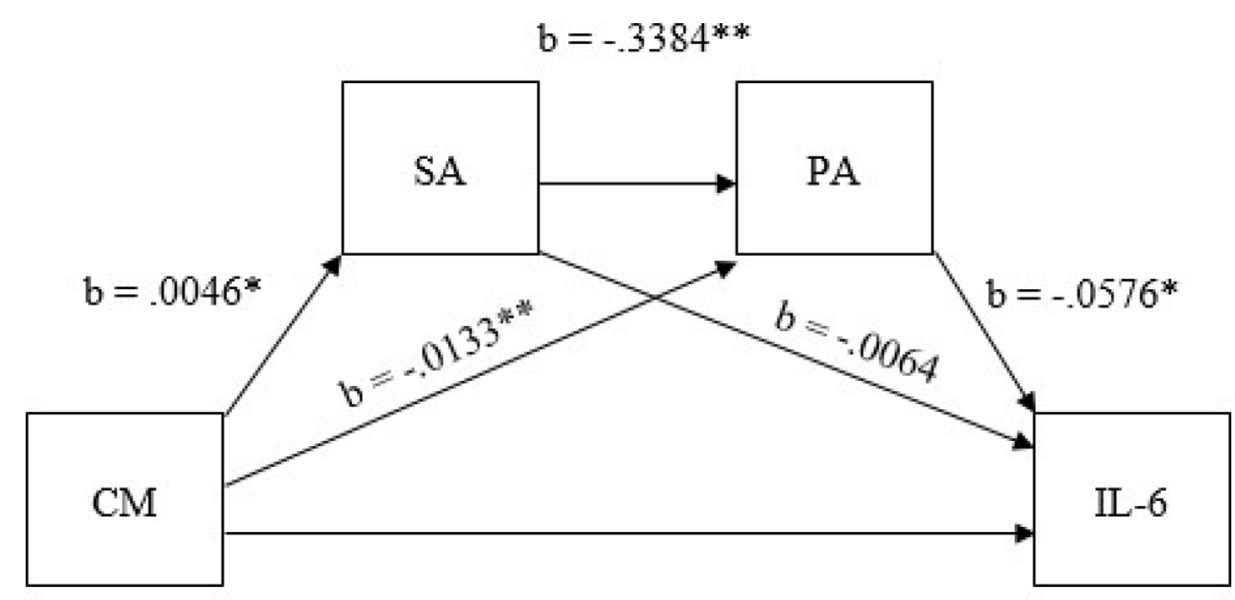

$\begin{array}{lc}\text { Total effect }(\mathrm{c}): & \mathrm{b}=.0024, \mathrm{SE}=.0010, \mathrm{p}=.0197 \\ \text { Direct effect }\left(\mathrm{c}^{\prime}\right): & \mathrm{b}=.0015, \mathrm{SE}=.0010, \mathrm{p}=.1393 \\ \text { Indirect effect }(\mathrm{ab}): & \mathrm{b}=.0008, \text { Boot SE }=.0004 \\ & \quad \text { Boot CI95\% }=.0002-.0017\end{array}$

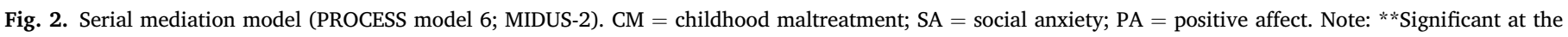
$p<.001$ level; * Significant at the $p<.01$ level.

(covarying NA over the total model), we conversely considered the influence of covarying PA over the total relationships, which examined the mediational status of NA in the indirect pathway from childhood maltreatment, to elevated IL-6. Results from this model indicated that childhood maltreatment was not significantly associated with social anxiety $(\mathrm{b}=.020, \mathrm{p}=.25)$ but was significantly related to $\mathrm{NA} \mathrm{b}=.003$, $\mathrm{p}<.05$ ), when controlling for PA. As was the case in the model covarying NA over the indirect path including PA, the direct effect of childhood maltreatment on IL- 6 was not significant $(b=.001, p=.21)$ when including PA over the indirect path including NA. As before, it is possible that this result is likely due to the strong (anti) correlation between PA and NA, thus leaving comparatively sparse variance available to drive the predicted associations. Results from this model indicated that childhood maltreatment was significantly and concurrently associated with both social anxiety symptoms and NA $(b=.005, p<.01 ; b=.010, p<.001$, respectively). The total effect of childhood maltreatment on IL-6 was significant $(b=.002, p<.05)$, and consistent with the presence of mediation, the direct effect was no longer significant after accounting for the presence of the indirect effect $(b=.002, p=.14)$. Scrutinizing individual paths in the model revealed that childhood maltreatment was significantly associated with social anxiety $(b=.005, p<.01)$, social anxiety next was significantly associated NA $(b=.326, p<.01)$, and NA was lastly associated with IL-6 $(b=.081, p<.01)$; however when considering the details of observed indirect effects, the full serial mediation effect (passing through both social anxiety and NA) was not significant (BootLLCI $=.0000$, BootULCI $=.0104$, containing zero); whereas the second mediated path (passing through only NA and bypassing social anxiety) was significant (BootLLCI $=.0081$, BootULCI $=$ .0591), suggestive of single or non-serial mediation in which the effect of child maltreatment on IL-6 bypasses social anxiety to indirectly impact IL-6 via NA.

\subsection{Conditional direct and indirect effects of trait mindfulness}

To further characterize the association between child maltreatment and IL- 6 , we examined the potential moderational status of trait mindfulness on the observed direct and indirect effects (moderated-mediation or conditional indirect and direct effect; PROCESS model 92). As before, results from this analysis indicated that childhood maltreatment was significantly associated with both social anxiety and PA $(b=.005$, $p<.01 ; b=-.014, p<.001$, respectively), and that social anxiety next predicted PA $(b=-.350, p<.001)$. Once again, consistent with the existence of full serial mediation, childhood maltreatment was not significantly associated with IL- 6 after accounting for the presence of an indirect effect $(b=.001, p=.39)$, and PA predicted IL-6 $(b=-.061$, $p<.01$ ). However, in this exploratory model which probed the potential moderating effect of mindfulness simultaneously on all possible paths, we found evidence indicating that trait mindfulness moderated two paths in the model, specifically moderating the relationship between PA and IL$6(b=-.005, p<.05)$ and the relationship between childhood maltreatment and PA $(b=.001, p<.05)$. This suggests that individual differences in trait mindfulness influenced the association between PA and circulating IL- 6 as well as the association between PA and childhood maltreatment. We further examined comparative effects of NA; this model was run again, substituting NA instead of PA. Consistent with model 6 (serial mediation), results again indicated that childhood maltreatment was significantly associated with both social anxiety and NA ( $b=.005, p \leq .01 ; b=.011, p<.001$, respectively), and that social anxiety further predicted NA $(b=.335, p<.001)$. Childhood maltreatment was not significantly associated with IL- $6(b=.001, p=.33)$, and NA predicted IL- $6(b=.095, p<.001)$. However, when examining the influence of trait mindfulness on all observed paths, we found no evidence of moderational effects of trait mindfulness on any path in the model, suggesting that trait mindfulness may suppress IL-6 levels but exclusively through its influence on positive but not NA.

\section{Discussion}

The current study examined associations among childhood maltreatment, concentrations of IL-6, social anxiety, and PA. Specifically, the current investigation tested the overall hypothesis that childhood maltreatment would be associated with IL-6 levels albeit indirectly and serially via social anxiety symptoms and low PA. Lastly, in exploratory analyses we examined the potential role of trait mindfulness as a moderator among the relations between childhood maltreatment, social anxiety, PA and IL-6. In total, results provided novel evidence for full 


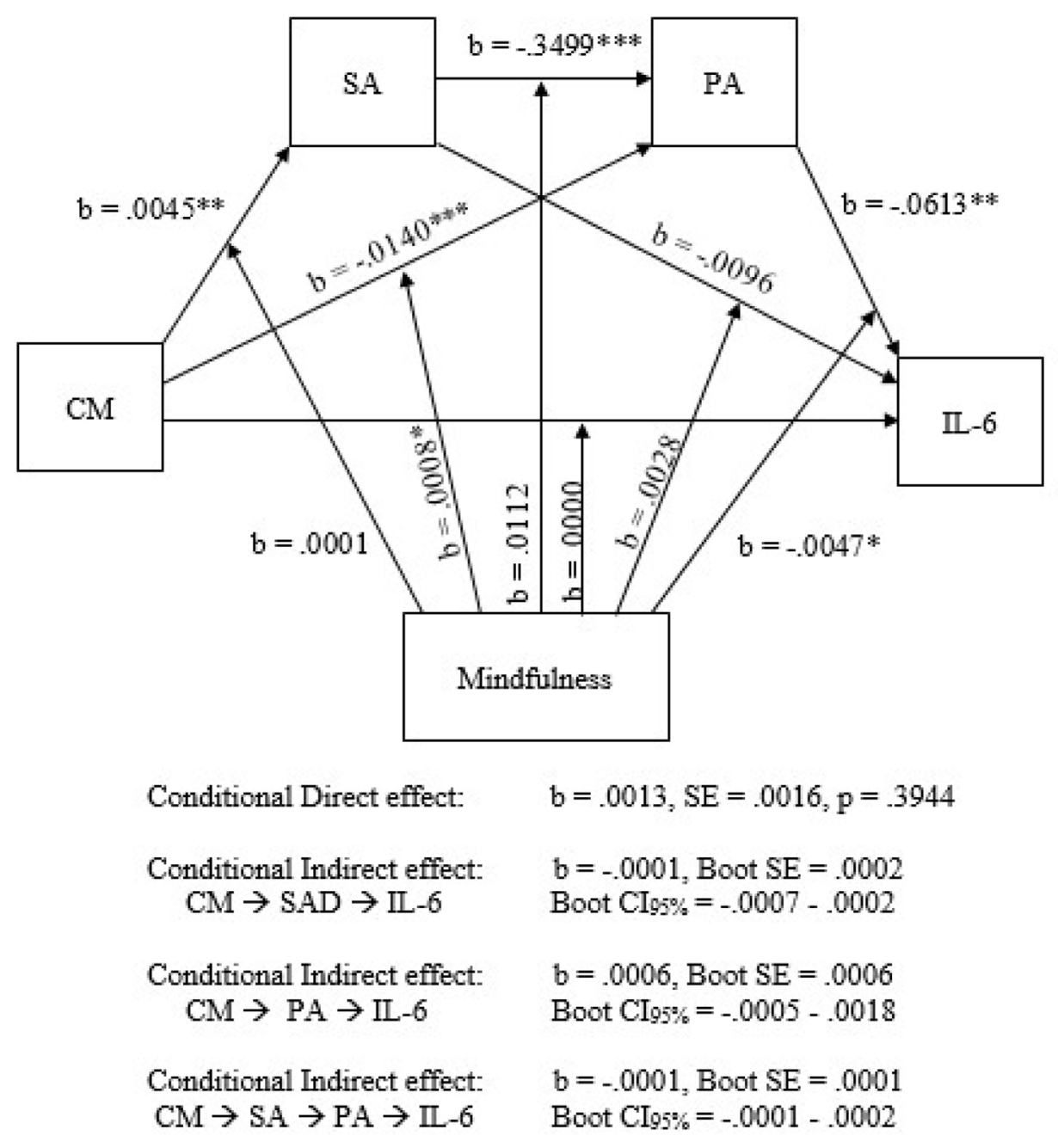

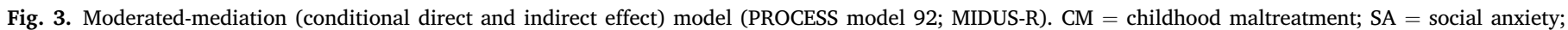
$\mathrm{PA}=$ positive affect. Note: ${ }^{* *}$ Significant at the $p<.001$ level; $* *$ Significant at the $p<.01$ level; * Significant at the $p<.05$ level.

serial mediation of the association between childhood maltreatment and IL- 6 by social anxiety severity and low PA and furthermore, when probing the same model utilizing NA instead of PA, results indicated that the association between childhood maltreatment and IL- 6 was mediated by NA, completely bypassing social anxiety. Lastly, results of exploratory analyses also indicated that the relation between PA and IL- 6 is moderated by trait mindfulness, however this association was not found in the path from NA to IL-6, suggesting that the relationship between PA and IL6 is potentially influenced by individual differences in trait mindfulness.

The current study advances the literature on the linkage between childhood maltreatment and adult health by identifying essential and potentially modifiable mechanisms that mediate and moderate the association between childhood maltreatment and inflammation in adulthood. Childhood maltreatment has been associated with a range of adverse health consequences in adulthood, as early experiences of abuse and neglect may become biologically relevant through their impact on systems that regulate metabolic function (see Hertzman and Boyce, 2010; Holochwost et al., 2020 for reviews). The identification of mediational factors such as those found here, imply that addressing social anxiety and also its negative sequelae such as low PA may ultimately impact inflammation in the context of childhood maltreatment. Specifically, inflammation and its own negative health consequences may be favorably impacted by directly engaging social anxiety among individuals endorsing high levels of early adversity as part of an understudied but evidently meaningful risk-chain, although future longitudinal research is necessary to replicate and confirm these pathways. Additionally, this study reinforces prior work on maltreatment and adult psychopathology by illustrating that overall childhood maltreatment experiences are associated with social anxiety symptoms. Similarly, our results reinforce prior findings on early adversity by showing that self-reported childhood maltreatment is also associated with proinflammatory biomarkers (e.g., Osborn and Widom, 2020).

Although literature exploring the nexus of social anxiety and inflammation is sparse, there is growing evidence to suggest that inflammation may be plausibly associated with social anxiety symptoms. Results within this literature are mixed however, with some studies reporting a demonstrable link between SAD and inflammation (e.g., Rapaport and Stein, 1994), in-line with findings reported here, whereas at least one study has not found such an effect (Vogelzangs et al., 2013). One potential explanation for this lack of identified association between social anxiety and inflammatory biomarkers reported by Vogelzangs et al. (2013) may in fact be due to the a priori assumption of linear effects, which, when applied to the data reported here, revealed a counterintuitive lack of association between social anxiety and IL-6. Consistent with results reported in Vogelzangs et al. (2013), we indeed found no linear association in the relation between social anxiety symptoms and IL-6 in the total sample $(F(1,319)=1.563, p=.212)$. However, further scrutiny of this relationship revealed evidence for a quadratic and not linear association between these variables. Further, consistent with the presence of a curvilinear association, we illustrated here that cases falling in the upper but not lower quartiles of social anxiety symptoms demonstrated the predicted significant linear relation between social anxiety symptoms 
and elevated concentrations of IL- 6 (upper quartile: $\beta=.28, \mathrm{p}<.05$; lower quartile: $\beta=-.18, p=.593$ ). The presence of this quadratic relation may be due to the specific association of higher severity social anxiety resulting in increased IL- 6 elevations, whereas this is may not be the case in milder symptom levels.

A significant body of research has supported the existence of relatively specific associations between low PA and social anxiety symptoms (Kashdan, 2007; Richey et al., 2017), and our findings extend this literature by highlighting the importance of social anxiety symptoms and low PA when examining childhood maltreatment and inflammation associations. Low levels of PA also are associated with some other forms of psychopathology such as depression, avoidant personality disorder (which overlaps closely with SAD; Carmichael et al., 2016), schizoid personality disorder (which includes anhedonia as a criterion), and negative symptoms of schizophrenia spectrum disorders (e.g., diminished emotional expression; Watson and Naragon-Gainey, 2014). Consequently, a profitable line of future inquiry involves the identification of meaningful linkages to other forms of psychopathology that follow from childhood maltreatment, particularly those that involve low PA. This is especially timely, given the National Institute of Mental Health's emphasis on characterizing how PA-related mechanisms and positively-valenced motivational states (e.g., reward responsivity, reward valuation) are implicated in the onset and maintenance of many symptom types via its Research Domain Criteria (RDoC) Initiative (Cuthbert, 2015). Similarly, from the perspective of the Hierarchical Taxonomy of Psychopathology (Kotov et al., 2017), there has been interest in linking broad psychopathology spectra such as internalizing (e.g., worry about judgment) and detachment (e.g., social withdrawal and aloofness) defined by features relevant to DSM-based conceptualizations of SAD to RDoC mechanisms (Perkins et al., 2020). The results from this study contribute to the growing identification of the extent to which associations observed may be specific to social anxiety symptomatology versus more transdiagnostic in nature, such that this present study identifies specific pathways through which social anxiety is influenced. Although SAD overlaps with other disorders characterized by low PA levels to varying degrees, prior meta-analytic research from Kashdan (2007; total $n=2976$ ) examining the relationship between PA and social anxiety symptoms across 19 studies still indicates a strong association for these constructs even after controlling for depressive symptoms and disorders. Although that particular meta-analytic approach was not focused on childhood maltreatment and inflammation, those aggregated results nevertheless highlight a noteworthy instance of specificity between social anxiety symptoms and low PA, above and beyond the symptoms of overlapping forms of psychopathology such as depression. Thus, social anxiety symptoms may indeed play an important role when examining associations between childhood maltreatment and inflammation even when accounting for other forms of affective psychopathology.

An additional incremental contribution of the work outlined here is the identification of trait mindfulness as a remarkably specific moderator of the relationship between low PA and IL- 6 and the relationship between childhood maltreatment and PA. Interest in mindfulness meditation as a method to reduce inflammation and improve physical health has grown substantially in recent years (Black and Slavich, 2016), motivated by seminal findings demonstrating the stress buffering effects of mindfulness training on neurogenic inflammation and the genetic mechanisms that subserve these processes (Buric et al., 2017). Various training programs have emerged in recent decades, most notably the Mindfulness-Based Stress Reduction (MBSR; Kabat-Zinn, 2003) protocol, among others such as Mindfulness-Based Cognitive therapy (Segal et al., 2018), which have been shown to reduce pro-inflammatory cytokines, such as IL-6, to reduce inflammation (e.g., Gallegos et al., 2015; Lengacher et al., 2019). Therefore, the present study provides an evidentiary basis for future work examining trait mindfulness as an intervention target for modulating inflammation among individuals with a history of low PA, and perhaps particularly so for individuals reporting a history of early adversity and/or social anxiety. Although, because this analysis was exploratory in nature here, these results should be both interpreted with caution and also further examined in a hypothesis testing framework in future research.

As with any study, results presented here should be evaluated in light of study limitations. First, childhood maltreatment experiences were assessed in the MIDUS-2 Biomarkers Project using retrospective selfreport and did not contain information about objectively rated experiences of abuse and neglect. However, self-report perceptions are a critical dimension of childhood maltreatment, and it has been demonstrated elsewhere that subjective self-reported childhood maltreatment relates more strongly to psychopathology than objective reports (Osborn and Widom, 2020). Nonetheless, it will be critical for future research to replicate findings reported here, using other measurement approaches and to consider a broader range of childhood maltreatment dimensions (such as chronicity, timing, distinct subtypes, etc.). Moreover, there has been debate in the field as to the application of mediational approaches in cross-sectional data (e.g., Maxwell and Cole, 2007), which lends the cross-sectional nature of the present data as a potential limitation. Thus, replication within longitudinal designs is critical before designing intervention strategies around the current results. Additionally, future longitudinal research should take a transdiagnostic perspective to consider mechanisms that may explain why childhood maltreatment is associated with social anxiety and other types of internalizing and externalizing psychopathology. Further, no specific timeline was provided for the elapsed time between PA and inflammation. Given that timing matters between this association (Graham-Engeland et al., 2018) future work should include clear timelines that collect momentary affect measures proximal to blood draw. An additional limitation that should be noted here is the exclusive reliance on a single marker of inflammatory activation, namely IL-6. Much of the rationale for focusing on a sole cytokine stems from the nascent nature of this work, which has not yet proliferated into mechanisms-focused explanations for the role of PA at the cellular and molecular levels (thus enabling strong hypotheses about specific roles for multifunctional cytokines). Future work may eventually have the benefit of detailed rationales for probing a multiplex or manifold approaches. However, we elected here to take a narrowly defined approach, partially based on the results of prior meta analysis which have supported the notion that pro-inflammatory cytokines such as TNF-alpha and IL- 6 are concurrently increased in serum of depressed patients (Dowlati et al., 2010). Also, we pursued this approach based on an assumption that a focus on IL-6 will facilitate future comparisons of the work presented here with prior work that has, in general, tended to focus on IL-6 as a principal mediator of general systemic inflammatory activation, immunity and disease (Xing, 1998; Tanaka et al., 2014). In light of this limitation, we explicitly suggest further hypothesis-driven work examining other markers of inflammatory activation in MIDUS and beyond. An additional limitation worthy of additional work lies in our finding that controlling for either PA/NA, mediation over indirect pats including NA/PA (respectively) were no longer significant. This is likely due to the strong (anti) correlation between PA and NA; however, this should be noted as a limitation to the present study and investigated further in future work. Lastly, we did not predict a nonlinear effect between social anxiety and IL-6; however, since a direct effect is not required for serially positioned mediational variables onto their respective dependent variable, we continued to investigate the relation between childhood maltreatment and IL- 6 as mediated by social anxiety and PA. Therefore, future work should probe this nonlinear association for its implications for treatment.

\section{Conclusion}

The present study demonstrated that the association between childhood maltreatment and IL- 6 is serially mediated by social anxiety levels and low PA, but not NA. Additionally, results indicated that the relation between IL- 6 and PA (but not NA) is moderated by trait mindfulness. 
Taken together, results of the current study identify potentially modifiable mechanisms that mediate and moderate the association between childhood maltreatment and inflammation in adulthood, suggesting that intervening to reduce social anxiety and improve PA has the potential to mitigate the long-term effects of childhood maltreatment on inflammation in adulthood. Moreover, results from the present study indicate that improvement in PA via improvements in mindfulness may be a particularly fruitful target for intervention.

\section{Funding statement}

This research did not receive any specific grant from funding agencies in the public, commercial, or not-for-profit sectors.

\section{Availability of data statement}

Both of the MIDUS datasets used in the present investigation are available on the Inter-University Consortium for Political and Social Research (ICPSR) website.

\section{Declaration of competing interest}

None.

\section{Acknowledgments}

Publically available data from two datasets in the MIDUS study was used for this research. The following funding sources should be acknowledged for their support of the MIDUS study since 1995: John D. and Catherine T. MacArthur Foundation Research Network; National Institute on Aging (P01-AG020166); National institute on Aging (U19AG051426). Biomarker data collection was further supported by the NIH National Center for Advancing Translational Sciences (NCATS), Clinical and Translational Science Award (CTSA) program as follows: UL1TR001409 (Georgetown), UL1TR001881 (UCLA), 1UL1RR025011 (UW).

\section{References}

American Psychiatric Association, 2013. Diagnostic and Statistical Manual of Mental Disorders, fifth ed. American Psychiatric Association, Arlington, VA.

Andreasson, A.N., Szulkin, R., Undén, A.L., Von Essen, J., Nilsson, L.G., Lekander, M. 2013. Inflammation and positive affect are associated with subjective health in women of the general population. J. Health Psychol. 18 (3), 311-320.

Arreola, R., Alvarez-Herrera, S., Pérez-Sánchez, G., Becerril-Villanueva, E., CruzFuentes, C., Flores-Gutierrez, E.O., et al., 2016. Immunomodulatory effects mediated by dopamine. J. Immun. Res. 2016.

Bandelow, B., Torrente, A.C., Wedekind, D., Broocks, A., Hajak, G., Rüther, E., 2004. Early traumatic life events, parental rearing styles, family history of mental disorders, and birth risk factors in patients with social anxiety disorder. Eur. Arch. Psychiatr. Clin. Neurosci. 254 (6), 397-405.

Barnett, D., Manly, J.T., Cicchetti, D., 1993. Defining Child Maltreatment: the Interface between Policy and Research. In: Cicchetti, D., Toth, S.L. (Eds.), Child Abuse, Child Development, and Social Policy. Ablex, Norwood, NJ, pp. 7-74.

Baumeister, D., Akhtar, R., Ciufolini, S., Pariante, C.M., Mondelli, V., 2016. Childhood trauma and adulthood inflammation: a meta-analysis of peripheral C-reactive protein, interleukin-6 and tumour necrosis factor- $\alpha$. Mol. Psychiatr. 21 (5), 642-649.

Beck, G.C., Brinkkoetter, P., Hanusch, C., Schulte, J., van Ackern, K., van der Woude, F.J., Yard, B.A., 2004. Clinical review: immunomodulatory effects of dopamine in general inflammation. Crit. Care 8 (6), 1-7.

Bell, J.A., Kivimäki, M., Bullmore, E.T., Steptoe, A., Carvalho, L.A., 2017. Repeated exposure to systemic inflammation and risk of new depressive symptoms among older adults. Transl. Psychiatry 7 (8) e1208-e1208.

Beshai, S., Parmar, P., 2019. Trait mindfulness may buffer against the deleterious effects of childhood abuse in recurrent depression: a retrospective exploratory study. Clin. Psychol. 23 (1), 26-36.

Berstein, D.P., Fink, L., 1998. Childhood Trauma Questionnaire: A Retrospective Self Report. The Psychological Corporation.

Bierhaus, A., Wolf, J., Andrassy, M., Rohleder, N., Humpert, P.M., Petrov, D., et al., 2003. A mechanism converting psychosocial stress into mononuclear cell activation. Proc. Natl. Acad. Sci. Unit. States Am. 100 (4), 1920-1925.

Black, D.S., Slavich, G.M., 2016. Mindfulness meditation and the immune system: a systematic review of randomized controlled trials. Ann. N. Y. Acad. Sci. 1373 (1), 13.
Bromberg-Martin, E.S., Matsumoto, M., Hikosaka, O., 2010. Dopamine in motivational control: rewarding, aversive, and alerting. Neuron 68 (5), 815-834. https://doi.org/ 10.1016/j.neuron.2010.11.022.

Brown, T.A., Chorpita, B.F., Barlow, D.H., 1998. Structural relationships among dimensions of the DSM-IV anxiety and mood disorders and dimensions of negative affect, positive affect, and autonomic arousal. J. Abnorm. Psychol. 107 (2), 179.

Bruce, L.C., Heimberg, R.G., Goldin, P.R., Gross, J.J., 2013. Childhood maltreatment and response to cognitive behavioral therapy among individuals with social anxiety disorder. Depress. Anxiety 30 (7), 662-669.

Buric, I., Farias, M., Jong, J., Mee, C., Brazil, I.A., 2017. What is the molecular signature of mind-body interventions? A systematic review of gene expression changes induced by meditation and related practices. Front. Immunol. 8, 670.

Carmichael, K.L.C., Sellbom, M., Ligget, L., Smith, A., 2016. A personality and impairment approach to examine the similarities and differences between avoidant personality disorder and social anxiety disorder. Pers. Ment. Health 10 (4), 337-347.

Carroll, J.E., Low, C.A., Prather, A.A., Cohen, S., Fury, J.M., Ross, D.C., Marsland, A.L. 2011. Negative affective responses to a speech task predict changes in interleukin (IL)-6. Brain Behav. Immun. 25 (2), 232-238.

Cicchetti, D., 2016. Socioemotional, personality, and biological development: illustrations from a multilevel developmental psychopathology perspective on child maltreatment. Annu. Rev. Psychol. 67, 187-211.

Cicchetti, D., Toth, S.L., 2005. Child maltreatment. Annu. Rev. Clin. Psychol. 1, 409-438.

Copeland, W.E., Shanahan, L., Worthman, C., Angold, A., Costello, E.J., 2012. Cumulative depression episodes predict later C-reactive protein levels: a prospective analysis. Biol. Psychiatr. 71 (1), 15-21.

Craske, M.G., Meuret, A.E., Ritz, T., Treanor, M., Dour, H.J., 2016. Treatment for anhedonia: a neuroscience driven approach. Depress. Anxiety 33 (10), 927-938.

Creswell, J.D., Taren, A.A., Lindsay, E.K., Greco, C.M., Gianaros, P.J., Fairgrieve, A., Marsland, A.L., Brown, K.W., Way, B.M., Rosen, R.K., Ferris, J.L., 2016. Alterations in resting-state functional connectivity link mindfulness meditation with reduced interleukin-6: a randomized controlled trial. Biol. Psychiatr. 80 (1), 53-61.

Cuthbert, B.N., 2015. Research Domain Criteria: toward future psychiatric nosologies. Dialogues Clin. Neurosci. 17 (1), 89.

Danese, A., Lewis, S.J., 2017. Psychoneuroimmunology of early-life stress: the hidden wounds of childhood trauma? Neuropsychopharmacology 42 (1), 99-114.

Danese, A., Moffitt, T.E., Pariante, C.M., Ambler, A., Poulton, R., Caspi, A., 2008. Elevated inflammation levels in depressed adults with a history of childhood maltreatment. Arch. Gen. Psychiatr. 65 (4), 409-415.

Danese, A., Pariante, C.M., Caspi, A., Taylor, A., Poulton, R., 2007. Childhood maltreatment predicts adult inflammation in a life-course study. Proc. Natl. Acad. Sci. Unit. States Am. 104 (4), 1319-1324.

Dantzer, R., O'Connor, J.C., Freund, G.G., Johnson, R.W., Kelley, K.W., 2008. From inflammation to sickness and depression: when the immune system subjugates the brain. Nat. Rev. Neurosci. 9 (1), 46-56.

De La Garza II, R., 2005. Endotoxin-or pro-inflammatory cytokine-induced sickness behavior as an animal model of depression: focus on anhedonia. Neurosci. Biobehav. Rev. 29 (4-5), 761-770.

DellaGioia, N., Hannestad, J., 2010. A critical review of human endotoxin administration as an experimental paradigm of depression. Neurosci. Biobehav. Rev. 34 (1), $130-143$.

Deverts, D.J., Cohen, S., DiLillo, V.G., Lewis, C.E., Kiefe, C., Whooley, M., Matthews, K.A., 2010. Depressive symptoms, race, and circulating C-reactive protein: the coronary artery risk development in young adults (CARDIA) study. Psychosom. Med. 72 (8), 734.

Dowlati, Y., Herrmann, N., Swardfager, W., Liu, H., Sham, L., Reim, E.K., Lanctôt, K.L., 2010. A meta-analysis of cytokines in major depression. Biol. Psychiatr. 67 (5), 446-457.

Dunlop, B.W., Nemeroff, C.B., 2007. The role of dopamine in the pathophysiology of depression. Arch. Gen. Psychiatr. 64 (3), 327-337. https://doi.org/10.1001/ archpsyc.64.3.327.

Dutcher, J.M., Boyle, C.C., Eisenberger, N.I., Cole, S.W., Bower, J.E., 2020. Neural responses to threat and reward and changes in inflammation following a mindfulness intervention. Psychoneuroendocrinology, 105114.

Duivis, H.E., de Jonge, P., Penninx, B.W., Na, B.Y., Cohen, B.E., Whooley, M.A., 2011. Depressive symptoms, health behaviors, and subsequent inflammation in patients with coronary heart disease: prospective findings from the heart and soul study. Am. J. Psychiatr. 168 (9), 913-920.

Eisenberger, N.I., Berkman, E.T., Inagaki, T.K., Rameson, L.T., Mashal, N.M., Irwin, M.R., 2010. Inflammation-induced anhedonia: endotoxin reduces ventral striatum responses to reward. Biol. Psychiatr. 68 (8), 748-754.

Euesden, J., Danese, A., Lewis, C.M., Maughan, B., 2017. A bidirectional relationship between depression and the autoimmune disorders-New perspectives from the National Child Development Study. PLoS One 12 (3), e0173015.

Fancourt, D., Steptoe, A., 2020. The longitudinal relationship between changes in wellbeing and inflammatory markers: are associations independent of depression? Brain Behav. Immun. 83, 146-152.

Felger, J.C., 2016. The role of dopamine in inflammation-associated depression: mechanisms and therapeutic implications. Inflammation-Associated Depression: Evidence, Mechanisms and Implications 199-219.

Felger, J.C., Lotrich, F.E., 2013. Inflammatory cytokines in depression: neurobiological mechanisms and therapeutic implications. Neuroscience 246, 199-229.

Felger, J.C., Miller, A.H., 2012. Cytokine effects on the basal ganglia and dopamine function: the subcortical source of inflammatory malaise. Front. Neuroendocrinol. 33 (3), 315-327. 
Friedman, E.M., Hayney, M., Love, G.D., Singer, B.H., Ryff, C.D., 2007. Plasma interleukin- 6 and soluble IL-6 receptors are associated with psychological well-being in aging women. Health Psychol. 26 (3), 305.

Fresco, D.M., Coles, M.E., Heimberg, R.G., Liebowitz, M.R., Hami, S., Stein, M.B., Goetz, D., 2001. The Liebowitz Social Anxiety Scale: a comparison of the psychometric properties of self-report and clinician-administered formats. Psychol. Med. 31 (6), 1025-1035.

Gallegos, A.M., Lytle, M.C., Moynihan, J.A., Talbot, N.L., 2015. Mindfulness-based stress reduction to enhance psychological functioning and improve inflammatory biomarkers in trauma-exposed women: a pilot study. Psychological Trauma: Theory, Research, Practice, and Policy 7 (6), 525.

Gardner, M.J., Thomas, H.J., Erskine, H.E., 2019. The association between five forms of child maltreatment and depressive and anxiety disorders: a systematic review and meta-analysis. Child Abuse Negl. 96, 104082.

Graham-Engeland, J.E., Sin, N.L., Smyth, J.M., Jones, D.R., Knight, E.L., Sliwinski, M.J., Almeida, D.M., Katz, M.J., Lipton, R.B., Engeland, C.G., 2018. Negative and positive affect as predictors of inflammation: timing matters. Brain Behav. Immun. 74, 222-230.

Hayes, A.F., 2012. PROCESS: A Versatile Computational Tool for Observed Variable Mediation, Moderation, and Conditional Process Modeling.

Hayes, A.F., Montoya, A.K., Rockwood, N.J., 2017. The analysis of mechanisms and their contingencies: PROCESS versus structural equation modeling. Australas. Mark. J. 25 (1), 76-81.

Hertzman, C., Boyce, T., 2010. How experience gets under the skin to create gradients in developmental health. Annu. Rev. Publ. Health 31, 329-347.

Holochwost, S.J., Wang, G., Kolacz, J., Mills-Koonce, W.R., Klika, J.B., Jaffee, S.R., 2020. The Neurophysiological Embedding of Child Maltreatment. Development and psychopathology, pp. 1-31.

Hunt, C.A., Smith, M.T., Mun, C.J., Irwin, M.R., Finan, P.H., 2021. Trait positive affect buffers the association between experimental sleep disruption and inflammation. Psychoneuroendocrinology 129, 105240.

Janicki-Deverts, D., Cohen, S., Doyle, W.J., Turner, R.B., Treanor, J.J., 2007. Infectioninduced proinflammatory cytokines are associated with decreases in positive affect, but not increases in negative affect. Brain Behav. Immun. 21 (3), 301-307.

Jislin-Goldberg, T., Tanay, G., Bernstein, A., 2012. Mindfulness and positive affect: crosssectional, prospective intervention, and real-time relations. J. Posit. Psychol. 7 (5), 349-361.

Kabat-Zinn, J., 2003. Mindfulness-based interventions in context: past, present, and future. Clin. Psychol. Sci. Pract. 10 (2), 144-156.

Kashdan, T.B., 2007. Social anxiety spectrum and diminished positive experiences: theoretical synthesis and meta-analysis. Clin. Psychol. Rev. 27 (3), 348-365.

Kashdan, T.B., Collins, R.L., 2010. Social anxiety and the experience of positive emotion and anger in everyday life: an ecological momentary assessment approach. Hist. Philos. Logic 23 (3), 259-272.

Kashdan, T.B., Steger, M.F., 2006. Expanding the topography of social anxiety: an experience-sampling assessment of positive emotions, positive events, and emotion suppression. Psychol. Sci. 17 (2), 120-128.

Kashdan, T.B., Weeks, J.W., Savostyanova, A.A., 2011. Whether, how, and when social anxiety shapes positive experiences and events: a self-regulatory framework and treatment implications. Clin. Psychol. Rev. 31 (5), 786-799.

Kessler, R.C., Chiu, W.T., Demler, O., Walters, E.E., 2005. Prevalence, severity, and comorbidity of 12-month DSM-IV disorders in the national comorbidity survey replication. Arch. Gen. Psychiatr. 62 (6), 617-627.

Kessler, R.C., Davis, C.G., Kendler, K.S., 1997. Childhood adversity and adult psychiatric disorder in the US National Comorbidity Survey. Psychol. Med. 27 (5), 1101-1119.

Kiecolt-Glaser, J.K., Derry, H.M., Fagundes, C.P., 2015. Inflammation: depression fans the flames and feasts on the heat. Am. J. Psychiatr. 172 (11), 1075-1091. https:// doi.org/10.1176/appi.ajp.2015.15020152.

Kiecolt-Glaser, J.K., Gouin, J.P., Weng, N.P., Malarkey, W.B., Beversdorf, D.Q., Glaser, R., 2011. Childhood adversity heightens the impact of later-life caregiving stress on telomere length and inflammation. Psychosom. Med. 73 (1), 16.

Kiecolt-Glaser, J.K., McGuire, L., Robles, T.F., Glaser, R., 2002. Psychoneuroimmunology: psychological influences on immune function and health. J. Consult. Clin. Psychol. 70 (3), 537.

Kotov, R., Krueger, R.F., Watson, D., Achenbach, T.M., Althoff, R.R., Bagby, R.M., Brown, T.A., Carpenter, W.T., Caspi, A., Clark, L.A., Eaton, N.R., Forbes, M.K., Forbush, K.T., Goldberg, D., Hasin, D., Hyman, S.E., Ivanova, M.Y., Lynam, D.R., Markon, K., Zimmerman, M., 2017. The Hierarchical Taxonomy of Psychopathology (HiTOP): a dimensional alternative to traditional nosologies. J. Abnorm. Psychol. 126 (4), 454-477.

Kudinova, A.Y., Gano, A., James, K.M., Lawlor, C., Deak, T., Gibb, B.E., 2020. Anhedonia and increased evoked immune response. Brain, Behav. Immunity-Health. 6. https:// doi.org/10.1016/j.bbih.2020.100090.

Kuebler, U., Zuccarella-Hackl, C., Arpagaus, A., Wolf, J.M., Farahmand, F., von Känel, R., et al., 2015. Stress-induced modulation of NF-кB activation, inflammation-associated gene expression, and cytokine levels in blood of healthy men. Brain Behav. Immun. 46, 87-95.

Kuo, J.R., Goldin, P.R., Werner, K., Heimberg, R.G., Gross, J.J., 2011. Childhood trauma and current psychological functioning in adults with social anxiety disorder. J. Anxiety Disord. 25 (4), 467-473.

Langer, E.J., Moldoveanu, M., 2000. The construct of mindfulness. J. Soc. Issues 56 (1), 129-139.

Lawrence, T., 2009. The nuclear factor B pathway in inflammation. Inflammation biology group. Cold Spring Harbor Laboratory Press 1 (6), 1-10.

Lengacher, C.A., Reich, R.R., Paterson, C.L., Shelton, M., Shivers, S., Ramesar, S., Pleasant, M.L., Budhrani-Shani, P., Groer, M., Post-White, J., Johnson-Mallard, V.,
Kane, B., Cousin, L., Moscoso, M.S., Romershausen, T.A., Park, J.Y., 2019. A large randomized trial: effects of mindfulness-based stress reduction (MBSR) for breast cancer (BC) survivors on salivary cortisol and IL-6. Biol. Res. Nurs. 21 (1), 39-49.

Liu, P.P., Xie, Y., Meng, X.Y., Kang, J.S., 2019. Signal Transduct. Target. Ther 4 (29), $10-1038$.

Makadi, E., Koszycki, D., 2020. Exploring connections between self-compassion, mindfulness, and social anxiety. Mindfulness 11 (2), 480-492.

Malarkey, W.B., Jarjoura, D., Klatt, M., 2013. Workplace based mindfulness practice and inflammation: a randomized trial. Brain Behav. Immun. 27, 145-154.

Matthews, K.A., Schott, L.L., Bromberger, J.T., Cyranowski, J.M., Everson-Rose, S.A., Sowers, M., 2010. Are there bi-directional associations between depressive symptoms and C-reactive protein in mid-life women? Brain Behav. Immun. 24 (1), 96-101.

Marsland, A.L., Walsh, C., Lockwood, K., John-Henderson, N.A., 2017. The effects of acute psychological stress on circulating and stimulated inflammatory markers: a systematic review and meta-analysis. Brain Behav. Immun. 64, 208-219.

Maxwell, S.E., Cole, D.A., 2007. Bias in cross-sectional analyses of longitudinal mediation. Psychol. Methods 12 (1), 23.

Mehta, N.D., Stevens, J.S., Li, Z., Gillespie, C.F., Fani, N., Michopoulos, V., Felger, J.C. 2020. Inflammation, reward circuitry and symptoms of anhedonia and PTSD in trauma-exposed women. Soc. Cognit. Affect Neurosci. 15 (10), 1046-1055.

Merikangas, K.R., He, J.P., Brody, D., Fisher, P.W., Bourdon, K., Koretz, D.S., 2010. Prevalence and treatment of mental disorders among US children in the 2001-2004 NHANES. Pediatrics 125, 75-81.

Michopoulos, V., Powers, A., Gillespie, C.F., Ressler, K.J., Jovanovic, T., 2017. Inflammation in fear-and anxiety-based disorders: PTSD, GAD, and beyond. Neuropsychopharmacology 42 (1), 254-270.

Miller, A.H., Maletic, V., Raison, C.L., 2009. Inflammation and its discontents: the role of cytokines in the pathophysiology of major depression. Biol. Psychiatr. 65 (9), 732-741.

Ng, T.K.S., Fam, J., Feng, L., Cheah, I.K.M., Tan, C.T.Y., Nur, F., Wee, S.T., Goh, L.G., Chow, W.L., Ho, R.C., Kua, E.H., Larbi, A., Kua, E.H., 2020. Mindfulness improves inflammatory biomarker levels in older adults with mild cognitive impairment: a randomized controlled trial. Transl. Psychiatry 10 (1), 1-14.

Nusslock, R., Miller, G.E., 2016. Early-life adversity and physical and emotional health across the lifespan: a neuroimmune network hypothesis. Biol. Psychiatr. 80 (1), 23-32.

O'Connor, M.F., Bower, J.E., Cho, H.J., Creswell, J.D., Dimitrov, S., Hamby, M.E., Hoyt, M.A., Martin, J.L., Robles, T.F., Sloan, E.K., Thomas, K.S., Irwin, M.R., 2009. To assess, to control, to exclude: effects of biobehavioral factors on circulating inflammatory markers. Brain Behav. Immun. 23 (7), 887-897.

Ong, A.D., Benson, L., Zautra, A.J., Ram, N., 2018. Emodiversity and biomarkers of inflammation. Emotion 18 (1), 3.

Osborn, M., Widom, C.S., 2020. Do documented records and retrospective reports of childhood maltreatment similarly predict chronic inflammation? Psychol. Med. 50 (14), 2406-2415.

Perkins, E.R., Patrick, C.J., Latzman, R.D., 2020. Interfacing neural constructs with the hierarchical Taxonomy of psychopathology: "why" and "how. Pers. Ment. Health 14 (1), 106-122.

Prather, A.A., Marsland, A.L., Muldoon, M.F., Manuck, S.B., 2007. Positive affective style covaries with stimulated IL-6 and IL-10 production in a middle-aged community sample. Brain Behav. Immun. 21 (8), 1033-1037.

Radler, B.T., 2014. The Midlife in the United States (MIDUS) series: a national longitudinal study of health and well-being. Open Health Data 2 (1).

Rapaport, M.H., Stein, M.B., 1994. Serum interleukin-2 and soluble interleukin-2 receptor levels in generalized social phobia. Anxiety 1 (2), 50-53.

Richey, J.A., Brewer, J.A., Sullivan-Toole, H., Strege, M.V., Kim-Spoon, J., White, S.W. Ollendick, T.H., 2019. Sensitivity shift theory: a developmental model of positive affect and motivational deficits in social anxiety disorder. Clin. Psychol. Rev. 72, 101756.

Richey, J.A., Ghane, M., Valdespino, A., Coffman, M.C., Strege, M.V., White, S.W., Ollendick, T.H., 2017. Spatiotemporal dissociation of brain activity underlying threat and reward in social anxiety disorder. Soc. Cognit. Affect Neurosci. 12 (1), 81-94.

Rosenkranz, M.A., Davidson, R.J., MacCoon, D.G., Sheridan, J.F., Kalin, N.H., Lutz, A., 2013. A comparison of mindfulness-based stress reduction and an active control in modulation of neurogenic inflammation. Brain Behav. Immun. 27, 174-184.

Rossetti, A.C., Papp, M., Gruca, P., Paladini, M.S., Racagni, G., Riva, M.A., Molteni, R., 2016. Stress-induced anhedonia is associated with the activation of the inflammatory system in the rat brain: restorative effect of pharmacological intervention. Pharmacol. Res. 103, 1-12.

Sanada, K., Montero-Marin, J., Barceló-Soler, A., Ikuse, D., Ota, M., Hirata, A., Yoshizawa, A., Hatanaka, R., Valero, M.S., Demarzo, M., Campayo, J.G., Iwanami, A., 2020. Effects of mindfulness-based interventions on biomarkers and low-grade inflammation in patients with psychiatric disorders: a meta-analytic review. Int. J. Mol. Sci. 21 (7), 2484.

Santambrogio, L., Lipartiti, M., Bruni, A., Dal Toso, R., 1993. Dopamine receptors on human T-and B-lymphocytes. J. Neuroimmunol. 45 (1-2), 113-119.

Segal, Z.V., Williams, M., Teasdale, J., 2018. Mindfulness-based Cognitive Therapy for Depression. Guilford Publications.

Simon, N.M., Herlands, N.N., Marks, E.H., Mancini, C., Letamendi, A., Li, Z., Pollack, M.H., Amerigen, M.V., Stein, M.B., 2009. Childhood maltreatment linked to greater symptom severity and poorer quality of life and function in social anxiety disorder. Depress. Anxiety 26 (11), 1027-1032.

Sin, N.L., Graham-Engeland, J.E., Almeida, D.M., 2015. Daily positive events and inflammation: findings from the national study of daily experiences. Brain Behav. Immun. 43, 130-138. 
Slavish, D.C., Jones, D.R., Smyth, J.M., Engeland, C.G., Song, S., McCormick, N.M., Graham-Engeland, J.E., 2020. Positive and negative affect and salivary markers of inflammation among young adults. Int. J. Behav. Med. 27 (3), 282-293.

Stellar, J.E., John-Henderson, N., Anderson, C.L., Gordon, A.M., McNeil, G.D., Keltner, D. 2015. Positive affect and markers of inflammation: discrete positive emotions predict lower levels of inflammatory cytokines. Emotion 15 (2), 129.

Steptoe, A., Gibson, E.L., Hamer, M., Wardle, J., 2007a. Neuroendocrine and cardiovascular correlates of positive affect measured by ecological momentary assessment and by questionnaire. Psychoneuroendocrinology 32 (1), 56-64.

Steptoe, A., Hamer, M., Chida, Y., 2007b. The effects of acute psychological stress on circulating inflammatory factors in humans: a review and meta-analysis. Brain Behav. Immun. 21 (7), 901-912.

Steptoe, A., O'Donnell, K., Marmot, M., Wardle, J., 2008. Positive affect, psychological well-being, and good sleep. J. Psychosom. Res. 64 (4), 409-415.

Steptoe, A., Wardle, J., Marmot, M., 2005. Positive affect and health-related neuroendocrine, cardiovascular, and inflammatory processes. Proc. Natl. Acad. Sci. Unit. States Am. 102 (18), 6508-6512.

Sturgeon, J.A., Finan, P.H., Zautra, A.J., 2016. Affective disturbance in rheumatoid arthritis: psychological and disease-related pathways. Nat. Rev. Rheumatol. 12 (9), 532-542.

Swardfager, W., Rosenblat, J.D., Benlamri, M., McIntyre, R.S., 2016. Mapping inflammation onto mood: inflammatory mediators of anhedonia. Neurosci. Biobehav. Rev. 64, 148-166.

Sutin, A.R., Terracciano, A., Deiana, B., Naitza, S., Ferrucci, L., Uda, M., et al., 2010. High neuroticism and low conscientiousness are associated with interleukin-6. Psychol. Med. 40 (9), 1485-1493.
Taillieu, T.L., Brownridge, D.A., Sareen, J., Afifi, T.O., 2016. Childhood emotional maltreatment and mental disorders: results from a nationally representative adult sample from the United States. Child Abuse Negl. 59, 1-12.

Tak, P.P., Firestein, G.S., 2001. NF-kB: a key role in inflammatory diseases. J. Clin. Invest. 107 (1), 7-11.

Tanaka, T., Narazaki, M., Kishimoto, T., 2014. IL-6 en la inflamación, la inmunidad, y la enfermedad. Cold Spring Harbor Perspectivas de la Biología 6 (10), a016295.

Villalba, D.K., Lindsay, E.K., Marsland, A.L., Greco, C.M., Young, S., Brown, K.W., Smyth, J.M., Walsh, C.P., Gray, K., Chin, B., Creswell, J.D., 2019. Mindfulness training and systemic low-grade inflammation in stressed community adults: evidence from two randomized controlled trials. PLoS One 14 (7), e0219120.

Vogelzangs, N., Beekman, A.T.F., De Jonge, P., Penninx, B.W.J.H., 2013. Anxiety disorders and inflammation in a large adult cohort. Transl. Psychiatry 3 (4) e249e249.

Watson, D., Clark, L.A., Tellegen, A., 1988. Development and validation of brief measures of positive and negative affect: the PANAS scales. J. Pers. Soc. Psychol. 54 (6), 1063.

Watson, D., Naragon-Gainey, K., 2014. Personality, emotions, and the emotional disorders. Clinical Psychological Science 2 (4), 422-442.

Xing, Z., 1998. Enhanced acute inflammatory responses in IL6-deficient mice IL6 is an antiinflammatory cytokine required for controlling local or systemic acute inflammatory responses. J. Clin. Invest. 101, 311-320.

Yadid, G., Friedman, A., 2008. Dynamics of the dopaminergic system as a key component to the understanding of depression. Prog. Brain Res. 172, 265-286.

Yoshioka, Y., Sugino, Y., Shibagaki, F., Yamamuro, A., Ishimaru, Y., Maeda, S., 2020. Dopamine attenuates lipopolysaccharide-induced expression of proinflammatory cytokines by inhibiting the nuclear translocation of NF-kB p65 through the formation of dopamine quinone in microglia. Eur. J. Pharmacol. 866, 172826. 